\title{
EVALUACIÓN DEL CONTENIDO DE POLIFENOLES TOTALES Y LA CAPACIDAD ANTIOXIDANTE DE LOS EXTRACTOS ETANÓLICOS DE LOS FRUTOS DE AGUAYMANTO (Physalis peruviana L.) DE DIFERENTES LUGARES DEL PERÚ
}

\author{
Bertha Jurado Teixeira $^{1 *}$, Isabel Mercedes Aparcana Ataurima ${ }^{1}$, \\ Leydi Steffani Villarreal Inca ${ }^{1}$, Eva Ramos Llica ${ }^{1}$, María Rosario Calixto Cotos ${ }^{2}$, \\ Paola Estefanía Hurtado Manrique ${ }^{1}$, Katherin María del Carmen Acosta Alfaro ${ }^{1}$
}

\begin{abstract}
RESUMEN
El aguaymanto es una especie nativa de la serranía peruana, que posee un diminuto fruto que discrepa con su grandeza nutricional y terapéutica. La presente investigación tuvo como objetivos valorar y comparar el contenido de polifenoles totales por el método de FolinCiocalteu y la capacidad antioxidante de los frutos de Physalis peruviana L., provenientes de Huánuco, Junín, Ancash y Cajamarca, por el método del DPPH (1,1-difenil-2-picrilhidrazilo). El fruto procedente de Huánuco presentó mayor contenido de compuestos fenólicos expresados como 149,3 $\pm 1,62 \mathrm{mg} / \mathrm{Eq}$ de ácido gálico/100 g de fruto. Asimismo, mayor capacidad antioxidante obteniendo como concentración inhibitoria $\mathrm{IC}_{50} 1,86 \mathrm{mg} / \mathrm{mL}$, comparado con los frutos provenientes de Junín, Ancash y Cajamarca; por lo que resultaría una buena fuente para la elaboración de diversos productos alimenticios benéficos para la salud (funcionales o nutraceúticos) y para la economía.
\end{abstract}

Palabras clave: Physalis peruviana L., polifenoles, actividad antioxidante.

\section{EVALUATION OF THE CONTENT OF PHENOLIC COMPOUNDS AND ANTIOXIDANT CAPACITY OF THE ETHANOL EXTRACTS OF THE FRUITS OF AGUAYMANTO (Physalis peruviana L.) FROM DIFFERENT LOCATIONS OF PERU}

\begin{abstract}
The aguaymanto is a native species of the Peruvian highland, which has a tiny fruit that disagrees with its greatness and nutritional therapy. The present investigation had as objetive to assess and compare the total polyphenol content by the Folin-Ciocalteu method and

\footnotetext{
1. Laboratorio de Farmacognosia y Medicina Tradicional. Instituto de Investigación en Ciencias Farmacéuticas y Recursos Naturales "Juan de Dios Guevara". Facultad de Farmacia y Bioquímica. Universidad Nacional Mayor de San Marcos. Jr Puno 1002. Jardín Botánico. Lima.*bjurado7@hotmail.com

${ }^{2}$ Instituto de Investigación de Ciencias Químicas. Facultad de Química e Ingeniería Química. Universidad Nacional Mayor de San Marcos.
} 
antioxidant capacity of the fruits of Physalis peruviana L., from Huánuco, Junín, Ancash y Cajamarca by the method of the DPPH (1,1-diphenyl-2-picril-hidrazilo). The fruit coming from Huánuco presented a higher content of phenolic compounds expressed as $149.3 \pm$ $1.62 \mathrm{mg} / \mathrm{Eq}$ of gallic acid/100g fruit. In addition, greater antioxidant capacity obtaining as inhibitory concentration IC50 $1.86 \mathrm{mg} / \mathrm{mL}$, compared with the fruits from Junín, Ancash y Cajamarca; and it would be a good source of consumption in benefit for the health.

Key words: Physalis peruviana L., polyphenols, antioxidant.

\section{INTRODUCCIÓN}

El Perú es un país privilegiado con enorme variedad de productos naturales. Como representante, tenemos un diminuto fruto proveniente de la serranía de nuestro país, de no más de dos centímetros, de color amarillo e impregnado de una brillantez y fragancia envidiable que está recubierto con un cáliz de cinco sépalos que le otorgan protección natural, llamado aguaymanto (Physalis peruviana L.) ${ }^{1}$.

Physalis peruviana L., pertenece a la familia Solanaceae, es una planta herbácea semiarbustiva y perenne que se caracteriza por tener raíces fibrosas y pivotantes, flores de cinco pétalos amarillos, hojas acorazonadas de color verde y tallo con vellosidades verdes de crecimiento lateral $^{2}$.

Numerosas publicaciones confieren a la especie propiedades beneficiosas para el ser humano, tanto medicinales como nutricionales. Tiene propiedad antiinflamatoria, hipoglicemiante, antihepatotóxica, antioxidante, tonifica el nervio óptico y purifica la sangre ${ }^{3-5}$. El fruto contiene compuestos bioactivos como polifenoles, provitamina A (3.000 IU de caroteno por $100 \mathrm{~g})$, vitamina C (20-43mg por $100 \mathrm{~g})$, vitamina $\mathrm{E}(86,30 \mathrm{~g}$ por kilo de lípidos totales, como el $\alpha$-tocoferol que elimina las especies reactivas de oxígeno $)^{6}$ y algunos complejos de vitamina B (tiamina, Riboflavina, niacina). Además, la fibra $(4,8 \%)$, proteína $(0,3 \%)$ y fósforo $(55 \%)$ contenido en esta baya son excepcionalmente altos para esta fruta ${ }^{3-5}$.

Los compuestos fenólicos constituyen uno de los más numerosos y representativos grupos de metabolitos secundarios de las plantas y su relevancia radica en su participación en la fisiología y el metabolismo celular como morfología, crecimiento, reproducción, defensa contra plagas y depredadores, y procesos germinativos, entre otros. Estos compuestos están presentes en la mayoría de los productos naturales consumidos por el hombre y en estudios recientes se ha demostrado una significativa actividad antioxidante, que evidencia su potencial benéfico sobre la salud humana ${ }^{7}$.

En un estudio se evaluó el contenido de compuestos fenólicos y la capacidad antioxidante de Physalis peruviana L., encontrándose mayor cantidad de estos compuestos, según el método de Folin-Ciocalteu, en un extracto etanólico que en un extracto acuoso, reportando valores en un rango desde $57,9 \mathrm{mg} / \mathrm{Eq}$ de ácido gálico/100g de fruto hasta 90,80 $\mathrm{mg} / \mathrm{Eq}$ de ácido gálico/100g de fruto $^{8}$. 
Considerando el alto contenido de polifenoles, vitamina A, C, E; en un estudio de actividad antioxidante se reportó un valor de $60 \%$ de inhibición del radical libre DPPH+ (1,1-difenil-2picril-hidrazilo) en un extracto etanólico del fruto a una concentración $60 \mathrm{mg} / \mathrm{dL}$, y asimismo se expresó en un valor de 23,94 $\mu \mathrm{mol}$ Eq de ácido ascórbico/g de extracto 9 .

Los objetivos de la presente investigación fueron: evaluación del contenido de polifenoles totales por el método de Folin Ciocalteu y la actividad antioxidante mediante el método de inhibición del DPPH (1,1-difenil-2-picril-hidrazilo) del fruto de Physalis peruviana L. proveniente de Cajamarca, Ancash, Huánuco y Junín.

\section{PARTE EXPERIMENTAL}

Lugar de ejecución: El presente trabajo de investigación se realizó en el laboratorio de Farmacognosia de la Facultad de Farmacia y Bioquímica de la Universidad Nacional Mayor de San Marcos.

Muestra: Los frutos de aguaymanto (Physalis peruviana L.) se recolectaron de diferentes regiones del Perú: Ancash, Junín, Cajamarca y Huánuco; en un estado de madurez tres, según la NTC 458010 y en época de verano. La identificación de la planta fue realizada por el biólogo José Campos De la Cruz (C.B.P. 3796) en el Museo de Historia Natural de la Universidad Nacional Mayor de San Marcos (UNMSM).

Reactivos: Etanol absoluto QP, metanol QP, agua bidestilada, DPPH (2,2-difenil-1picrilhidrazil), trolox (6-hidroxi-2, 5, 7, 8 tetrametilcromo-2 ácido carboxílico 97\%) (SigmaAldrich USA), vitamina C (Sigma-Aldrich, USA), ácido gálico (Merck) y reactivo de FolinCiocalteu (Sigma-Aldrich, USA).

Equipos: Espectrofotómetro UV-visible (Merck), balanza analítica de capacidad de $1 \mathrm{mg}$ $200 \mathrm{~g}$ (Ohaus Pioneer), Estufa (Memmert).

Obtención del extracto de Physalis peruviana L.: Se seleccionaron los frutos sanos y firmes, se procedió a lavarlos con agua destilada y luego fueron licuados durante cinco minutos. Se pesaron 850 g de cada muestra, se maceró con $850 \mathrm{~mL}$ de etanol al $96 \%$ en un frasco de vidrio ámbar y se agitaron durante 15 minutos para luego ser almacenados a temperatura ambiente en oscuridad durante siete días. Posteriormente, se filtraron con papel filtro Whatman $40 \mathrm{y}$ se concentraron en una estufa a $40^{\circ} \mathrm{C}$ por tres días.

Determinación de compuestos fenólicos: Se utilizó el método de Folin-Ciocalteu descrito por Singleton et al. ${ }^{11}$. Se preparó una curva de calibración de ácido gálico cuyo rango de concentración fue de 1-7,5 $\mu \mathrm{g} / \mathrm{mL}$. Los extractos etanólicos de las cuatro muestras vegetales fueron evaluados a una concentración de $0,9 \mathrm{mg} / \mathrm{mL}$. A $0,3 \mathrm{~mL}$ de la muestra se le añadió $0,45 \mathrm{~mL}$ de la solución de Folin-Ciocalteu (diluido 1 en 2 con agua bidestilada) y se dejó reposar durante cinco minutos, luego se adicionó $0,45 \mathrm{~mL}$ de $\mathrm{Na}_{2} \mathrm{CO}_{3} 20 \%$ y posteriormente 
agua bidestilada hasta un volumen total de $3 \mathrm{~mL}$. Se agitó vigorosamente, se cubrió de la luz y se le llevó a reposo por 30 minutos a temperatura ambiente. Las absorbancias respectivas fueron medidas a $760 \eta \mathrm{m}$ en un espectrofotómetro. Las muestras fueron analizadas por triplicado y el contenido de compuestos fenólicos totales fue expresado en mg de ácido gálico/g de extracto etanólico.

Determinación de la actividad antioxidante: Se evaluó la actividad antioxidante por el método del DDPH descrita por Brand-Williams et al. ${ }^{12}$. Se preparó diluciones en agua bidestilada de los extractos etanólicos hasta obtener concentraciones de 0,8 a $4 \mathrm{mg} / \mathrm{mL}$. Se mezcló $0,8 \mathrm{~mL}$ de cada una de las diluciones con $1,6 \mathrm{~mL}$ de una solución de $20 \mathrm{mg} / \mathrm{L}$ de DPPH en metanol y se dejó reaccionar a temperatura ambiente por 30 minutos, al término de los cuales se procedió a medir la absorbancia de la mezcla a $517 \mathrm{~nm}$. Las muestras fueron analizadas por triplicado. Los resultados se expresaron como valores de porcentaje de capacidad antioxidante y de concentración media inhibitoria $\left(\mathrm{IC}_{50}\right)$.

$\%$ Capacidad antioxidante $=\frac{[1-((\text { Abs. Muestra con DPPH })-\text { Abs. Blanco muestra })]}{\text { Abs. } \mathrm{DPPH}^{+}} \times 100$

\section{RESULTADOS Y DISCUSIÓN}

En la figura 1 se observa la curva de calibración del ácido gálico usado como patrón de referencia para la determinación de compuestos fenólicos. En la tabla 1 se muestran los resultados de la cuantificación de fenoles totales por el método de Folin-Ciocalteu, cuyos resultados se expresan en $\mathrm{mg} / \mathrm{Eq}$ de ácido gálico/100g de fruto. Se observa que el extracto etanólico del fruto proveniente de Huánuco presentó mayor cantidad de fenoles totales $(149,3 \pm 1,62 \mathrm{mg} / \mathrm{Eq}$. ácido gálico/100g de fruto), valor superior al reportado por Mier et al. $(2011)^{13}$ de $131,19 \pm 0,0 \mathrm{mg} / \mathrm{Eq}$ ácido gálico/100g de fruto proveniente de Colombia en un estado de madurez tres. Según Repo y Encina $(2008)^{14}$ se obtuvo un valor de $154 \mathrm{mg} /$ Eq ácido gálico/100g de fruto proveniente de Cajamarca, superior a nuestro fruto analizado proveniente de la misma región; debido a que ellos han trabajado con un extracto metanólico y con frutos maduros; ya que el estado de madurez influye directamente en el contenido de compuestos fenólicos del aguaymanto. Es importante mencionar que los compuestos fenólicos que se encuentran en las frutas y vegetales, tienen un rol fundamental en la salud; ya que disminuyen el riesgo de enfermedades crónicas, tales como la enfermedad cardiovascular y cáncer (Boyer y Liu, 2004) ${ }^{15}$. 


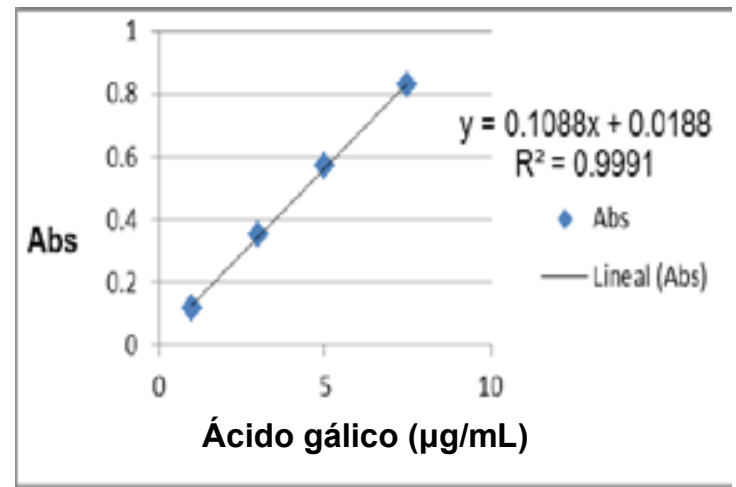

Figura 1. Curva de calibración del ácido gálico para la determinación de fenoles totales por el método de Folin-Ciocalteu

Tabla 1. Contenido de fenoles totales en los frutos de Physalis peruviana $\mathrm{L}$.

\begin{tabular}{cc}
\hline $\begin{array}{c}\text { Extracto seco de Physalis peruviana } \mathrm{L} . \\
\text { proveniente de: }\end{array}$ & $\begin{array}{c}\text { Fenoles totales }(\mathrm{mg} / \text { Eq. ácido } \\
\text { gálico/100g de fruto }\end{array}$ \\
\hline Huánuco & $149,3 \pm 1,62$ \\
Junín & $144,4 \pm 0,97$ \\
Ancash & $127,9 \pm 0,79$ \\
Cajamarca & $106 \pm 0,48$ \\
\hline
\end{tabular}

En la tabla 2 se observa los porcentajes de actividad antioxidante por el método DPPH de los frutos de aguaymanto, a diferentes concentraciones en un rango de 0,8 hasta $4 \mathrm{mg} / \mathrm{mL}$, el cual indica que el fruto proveniente de Huánuco presentó mayor porcentaje de captación de radicales libres $(100 \pm 1,72)$, seguido por los frutos provenientes de Junín, Ancash y Cajamarca; valor superior al reportado por Repo y Encina et al. $(2008)^{14}$ de $52 \%$ comparado con otras frutas nativas peruanas; debido a que ellos trabajaron con otra metodología de extracción, usando como solvente el metanol, tiempo de 24 horas y temperatura de $4^{\circ} \mathrm{C}$. Cabe mencionar que los factores de extracción como temperatura, tiempo y tipo de solvente influyen directamente en la determinación de los compuestos bioactivos ${ }^{16}$. Según Mier et al. $(2011)^{13}$ se obtuvo un valor de 79,61 $\pm 0,10 \%$ de captación de radicales DPPH del fruto de aguaymanto proveniente de Colombia, en un estado de madurez tres, valor cercano al obtenido en la muestra proveniente de Huánuco. Señalando, que el estado de madurez de un fruto influye directamente en el contenido de compuestos bioactivos, los cuales actúan en forma sinérgica para potenciar el efecto antioxidante. 
Tabla 2. Porcentaje de captación de radicales libres por el método DPPH

\begin{tabular}{ccccc}
\hline \multirow{2}{*}{$\begin{array}{c}\text { Conc. } \\
\mathrm{mg} / \mathrm{mL}\end{array}$} & \multicolumn{4}{c}{ \% Captación de radicales libres de Physalis peruviana L. } \\
\cline { 2 - 5 } & $100 \pm 1,72$ & $93,26 \pm 0,61$ & $86,86 \pm 0,28$ & $82,51 \pm 1,72$ \\
\hline 4 & Junín & Ancash & Cajamarca \\
3,2 & $96,98 \pm 0,76$ & $85,64 \pm 0,53$ & $83,57 \pm 0,18$ & $78,27 \pm 1,02$ \\
2,4 & $77,03 \pm 0,85$ & $73,56 \pm 0,45$ & $72,81 \pm 0,46$ & $56,81 \pm 0,37$ \\
1,6 & $67,59 \pm 1,67$ & $58,86 \pm 0,28$ & $58,68 \pm 1,56$ & $37,47 \pm 0,95$ \\
0,8 & $42,22 \pm 1,13$ & $30,95 \pm 0,11$ & $37,16 \pm 0,12$ & $25,55 \pm 1,92$ \\
\hline
\end{tabular}

En la tabla 3 se muestra los resultados de la evaluación de la actividad antioxidante y determinación del IC50 en los frutos de aguaymanto. El IC50 se define como la concentración necesaria de la muestra para disminuir en un 50\% la concentración inicial de los radicales libres (DPPH+), teniendo en cuenta que a menor valor de IC50 es mayor la actividad antioxidante17. Se observa que el fruto de Huánuco tuvo mayor porcentaje de captación de radicales libres y menor valor de IC50 $(1,86 \mathrm{mg} / \mathrm{mL})$.

Tabla 2. Porcentajes de captación de radicales y valores de $\mathrm{IC}_{50}(\mathrm{mg} / \mathrm{mL})$

\begin{tabular}{ccc}
\hline $\begin{array}{c}\text { Extracto de } \text { Physalis } \\
\text { peruviana } \mathrm{L} . \quad(2,4 \mathrm{mg} /\end{array}$ & $\begin{array}{c}\text { Porcentaje de } \\
\text { captación de } \\
\text { radicales (\%) }\end{array}$ & $\mathrm{IC}_{50}(\mathrm{mg} / \mathrm{mL})$ \\
\hline Huánuco & $77,03 \pm 0,85$ & 1,86 \\
Junín & $73,56 \pm 0,45$ & 2,04 \\
Ancash & $72,81 \pm 0,46$ & 2,24 \\
Cajamarca & $56,81 \pm 0,37$ & 2,36 \\
\hline
\end{tabular}

\section{CONCLUSIONES}

La determinación de fenoles totales fue de $149,3 \pm 1,62 ; 144,4 \pm 0,97 ; 127,9 \pm 0,79$ y $106 \pm$ 0,48 mg/Eq. AG/100 g fruto; correspondiendo estos a Huánuco, Junín, Ancash y Cajamarca, respectivamente, mostrándose el extracto etanólico de Huánuco con mayor cantidad de fenoles totales.

Los extractos etanólicos de Physalis peruviana L. de Huánuco, Junín, Ancash y Cajamarca, evaluados por el método del DPPH, expresaron un porcentaje de captación de radicales libres de DPPH de $100 \pm 1,72 ; 93,26 \pm 0,61 ; 86,86 \pm 0,28$ y $82,51 \pm 1,72 \%$, respectivamente; asimismo, expresaron un IC50 de 1,86;2,04; 2,24 y $2,36 \mathrm{mg} / \mathrm{mL}$, respectivamente; correspondiendo el de mayor capacidad antioxidante al extracto etanólico proveniente de Huánuco. 


\section{AGRADECIMIENTOS}

A la Facultad de Farmacia y Bioquímica de la Universidad Nacional Mayor de San Marcos.

\section{REFERENCIAS BIBLIOGRÁFICAS}

1. Ávalos C. Fruto Peruano que conquista el mundo. Generación [Internet]. Agosto 2010 [consultado 4 de septiembre 2014]; Disponible en:

http://www.generaccion.com/magazine/383/fruto-peruano-que-conquista-mundo

2. Trujillo JP. Aplicación de Elementos Intermitentes y Otros Coadyuvantes a la Conservación Frigorífica de Aguaymanto [Tesis Doctoral]. Madrid: Universidad Politécnica de Valencia, Facultad de Ciencias; 2007. 115 p.

3. Wu SJ, Tsai J, Chang SP, Lin DL, Wang S, Huang SN, et al. Supercritical carbon dioxide extracts exhibits enhanced antioxidant and anti-inflammatory activities of Physalis peruviana L. J Ethnopharmacol. 2006; 108(3): 407-413.

4. Franco LA, Matiz GE, Calle J, Pinzón R, Ospina LF, et al. Actividad antinflamatoria de extractos y fracciones obtenidas de cálices de Physalis peruviana L. Biomédica. 2007; 27(1): 110-115.

5. Pardo JM, Fontanilla MR, Ospina LF, Espinosa L. Determining the pharmacological activity of Physalis peruviana L. fruit juice on rabbit eyes and fibroblast primary cultures. Invest Ophthamol Vis Sci. 2008; 7(7): 3074-3079.

6. Puente LA, Pinto-Muñoz CA, Castro ES, Cortés M. Physalis peruviana L. Multiple properties of a highly functional fruit. Food Res Int. 2011; 44(7):1733-40. 2011.

7. Vidal A, Motidome M, Mancini J, Fallarero A, Midori M, et al. Actividad antioxidante y ácidos fenólicos del alga marina Bryothamnion triquetrum (S.G.Gmelim). Rev Bras Cienc Farm. 2001; 37 (3): 373-82.

8. Wu SJ, Tsai J, Chang SP, Lin DL, Wang S, Huang SN, et al. Supercritical carbon dioxide extracts exhibits enhanced antioxidant and anti-inflammatory activities of Physalis peruviana L. J Ethnopharmacol. 2006; 108: 407-413.

9. Zavaleta J, Muñoz AM, Blanco T, Alvarado-Ortiz C, Loja B. Capacidad antioxidante y principales ácidos fenólicos y flavonoides de algunos alimentos. Horiz Med. 2005; 2(5): 29-38.

10. ICONTEC. Frutas frescas. Uchuva. Especificaciones. Norma Técnica Colombiana NTC 4580. Bogotá: Instituto Colombiano de Normas Técnicas; 1999.

11. Singleton VL, Rossi JA. Colorimetry of total phenolics with phosphomolybdicphosphotungstic acid reagents. Am J Enol Vitic. 1965; 16:144-158.

12. Brand-Williams W, Cuvelier M, Berset C. Use of a free radical method to evaluate antioxidant activity. Food Sci Technol. 1995; 20: 25-30.

13. Mier HJ, Cáez G. Contenido de polifenoles, carotenos y capacidad antioxidante en frutos de uchuva (Physalis peruviana L.) en relación a su estado de maduración. ReCiTeIA. 2011; 11(1b): 104-115.

14. Repo R, Encina C. Determinación de la capacidad antioxidante y compuestos bioactivos de frutas nativas peruanas. Rev Soc Quím Per. 2008; 74(2): 108-124. 
15. Boyer J, Lui RH. Apple phytochemicals and their health benefits. Nutr J. 2004; 3: 1-15.

16. Silva Ch S. Optimización del proceso de extracción supercrítica de los polifenoles de la vaina de tara (Caesalpinia spinosa) [Tesis]. Santiago de Chile: Universidad de Chile; 2012. $77 \mathrm{p}$.

17. Zhu KX, Lian CX, Guo XN, Zhou HM. Antioxidant activities and total phenolic contents of various extracts from defatted wheat germ. Food Chem. 2011; 126: 1122-1126. 